\title{
QoS-Guaranteed User Association in HetNets via Semidefinite Relaxation
}

\author{
Hamza Umit Sokun, Ramy H. Gohary, Halim Yanikomeroglu \\ Department of Systems and Computer Engineering, Carleton University, Ottawa, Ontario, Canada \\ E-mail: \{husokun, gohary, halim\}@sce.carleton.ca
}

\begin{abstract}
The objective of this work is to determine a closeto-optimal user-to-base station (BS) association that maximizes the number of users served by the downlink of a heterogeneous network (HetNet). Such an association must not only ensure that the number of accommodated users is maximized but also that the network resources are efficiently utilized and the users' quality of service $(\mathrm{QoS})$ demands are met. In its simplest form, the optimization problem that underlies this association is combinatorial NP-hard and the difficulty of solving it is aggravated in HetNets by the disparity of transmit powers and BS computational capabilities. To find close-to-optimal user-to-BS associations, we develop a two-phase method based on semidefinite relaxation (SDR) with randomization, which is a powerful method for solving a class of combinatorial problems. Unlike the majority of other polynomial complexity techniques, SDR has a provable approximation accuracy. Numerical examples show that, in comparison with other user-to-BS association approaches, the one proposed herein enables more efficient utilization of resources and a significantly higher number of users to be accommodated.
\end{abstract}

Index Terms-Heterogeneous cellular networks, Gaussian randomization, convex optimization.

\section{INTRODUCTION}

Despite the ostensible ubiquity of conventional homogeneous cellular networks, their inherent limitations render them incapable of supporting the users' increasing demand for reliable communication of higher data rates. Such limitations include inefficient utilization of spectral resources, poor coverage and excessive power consumption [1]. To mitigate these limitations, cellular networks have evolved to include low-power pico and femto base stations (BSs). Incorporating such BSs to assist homogeneous conventional macro ones gives rise to the socalled heterogeneous networks (HetNets). These networks offer significant advantages over their conventional counterparts. For instance, HetNets are capable of: making efficient utilization of the spectrum available for communication [1]; reducing the overall network power consumption [2]; eliminating coverage holes; and, with proper user-to-BS association, accommodating more users in the system as will be shown herein. Despite the advantages of HetNets, the disparate transmit powers and computational capabilities of the underlying BSs render fair and resource-efficient user-to-BS association a serious challenge [3].

This work is supported in part by Huawei Canada Co., Ltd., in part by the Ontario Ministry of Economic Development and Innovation's ORF-RE (Ontario Research Fund - Research Excellence) program, and in part by a Discovery Grant of the Natural Sciences and Engineering Research Council (NSERC) of Canada.
To overcome user-to-BS association challenges in HetNets, several considerations must be taken into account: 1) the channel conditions between the users and the BSs; 2) the load condition of each BS; and 3) the quality-of-service (QoS) demanded by each user. A conventional approach to associating users to BSs is the one in which each user is associated to the BS with the maximum signal-to-interference-plus-noise ratio (max-SINR). This approach is relatively simple, but can lead to highly imbalanced loading of the BSs and does not guarantee users' QoS. Furthermore, the max-SINR approach does not account for disparities in the transmit powers and BS computational capabilities. Another approach for user-to-BS association is the one based on range expansion (RE) [4]. In that approach, the traffic from macro BSs is offloaded to lowpower BSs by adding a positive bias to the measured SINRs of low-power BSs. An advantage of this approach is that it does not depend on the relative location of users and BSs. However, a major weakness of it is the lack of theoretical guidance for choosing the biasing factor. Algorithms for determining userto-BS associations by finding approximate solutions to relaxed network utility maximization problems using dual pricing methods were proposed in [5] and [6] for the case when the number of users in the system is fixed. Those methods do not account for the QoS requirements and the associations that they yield are not necessarily binary. The latter feature implies that the number of BSs serving a particular user can be practically infeasible. In this paper, we consider the association of users to BSs in the downlink of a generic HetNet. Such an association must not only ensure that the number of accommodated users in the network is maximized but also that the network resources are efficiently utilized and the users' QoS demands are met. In its simplest form, this problem is combinatorial NP-hard and the difficulty of solving it in HetNets is aggravated by the disparity of transmit powers and BS computational capabilities; finding the optimal solution even for relatively small homogeneous systems is computationally prohibitive. To find close-to-optimal user-to-BS associations, we propose a method based on SDR with randomization, which is a powerful method for solving a class of combinatorial problems [7]. In the first phase of this method, a convex formulation is used to generate a covariance matrix and an upper bound on the maximum number of users that can be accommodated in a network with controlled resource consumption and ensured QoS. In the second phase, the covariance matrix obtained in the first phase is used to generate a population of random candidates of 
the solution. The candidate that yields the highest objective is used for associating users to BSs. Unlike other techniques with polynomial complexity, SDR with randomization has a provable approximation accuracy. Numerical results suggest that, in comparison with other user-to-BS association approaches, SDR with randomization enables the system to accommodate a significantly higher number of satisfied users.

\section{SySTEM MODEL}

We consider the downlink of the static two-tier HetNet shown in Figure 1 [5]. In such a HetNet, tier-1 consists of one or more macro BSs and tier- 2 consists of one or more pico BSs. The set of BSs is denoted by $B=\left\{1, \cdots, N_{B}\right\}$ and the set of users is denoted by $U=\left\{1, \cdots, N_{U}\right\}$. All BSs are assumed to

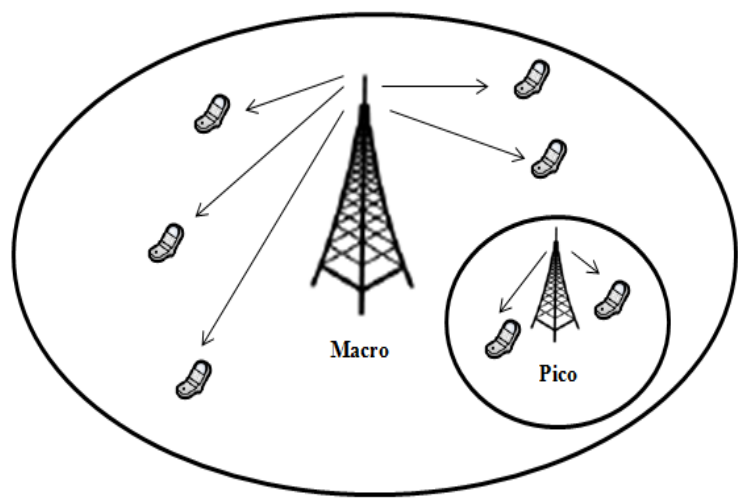

Fig. 1. A two-tier HetNet; macro BS transmits higher power than pico BS.

have full buffers and to have fixed transmit powers throughout the signalling interval. Using $g_{i j}$ to denote the channel gain between the $i$-th BS and the $j$-th user, $P_{i}$ to denote the transmit power of the $i$-th BS and $\sigma_{N}$ to denote the variance of the additive Gaussian noise, the received SINR observed by the $j$-th user from the $i$-th BS can be expressed as

$$
\gamma_{i j}=\frac{P_{i} g_{i j}}{\sum_{k \in B, k \neq i} P_{k} g_{k j}+\sigma_{N}}, \quad i \in B, j \in U .
$$

The corresponding rate at which the $i$-th $\mathrm{BS}$ can reliably communicate with the $j$-th user per unit bandwidth is

$$
\eta_{i j}=\log _{2}\left(1+\gamma_{i j}\right) \quad[\mathrm{bits} / \mathrm{s} / \mathrm{Hz}] .
$$

For practical considerations, many communication standards, including the current Long Term Evolution (LTE) one, impose a restriction on the smallest number of time-frequency resource blocks (RBs) that can be assigned to any user [8]. The number of RBs that must be assigned to a user depends on its QoS demand; higher QoS requirement implies a higher number of allocated RBs. To see that, let $Q_{j}$ be the data rate in bits/s required by the $j$-th user, $j=1, \ldots, N_{U}$, and let $W$ be the bandwidth of each RB. Using this notation, the minimum number of RBs that must be allocated by the $i$-th BS to meet the demand of the $j$-th user is given by

$$
b_{i j}=\left\lceil\frac{Q_{j}}{W \eta_{i j}}\right\rceil \text {, }
$$

where $[\cdot]$ is the ceiling function. From this expression, it can be seen that $b_{i j}$ is directly proportional to the QoS required by the $j$-th user and inversely proportional to the maximum per unit bandwidth rate that can be achieved on the $i j$-th link.

\section{Optimization Problem Formulation}

Using the characterization in Section II, we now develop an optimization framework for maximizing the number of users accommodated by the network while ensuring efficient utilization of the available resources. Towards that end, we begin by defining the binary association variables $x_{i j} \in\{0,1\}$ such that $x_{i j}=1$ if the $j$-th user is served by the $i$-th BS and $x_{i j}=0$ otherwise, $i=1, \ldots, N_{B}, j=1, \ldots, N_{U}$.

Using this notation, the total number of resources used by the $i$-th BS can be expressed as $\sum_{j \in U} b_{i j} x_{i j}$ and the total number of resources used in the network can be expressed as $\sum_{i \in B} \sum_{j \in U} b_{i j} x_{i j}$. To meet the constraints of the macro and pico BSs, a practically feasible design must ensure that the total number of resources used by the $i$-th BS does not exceed a given threshold $t_{i}$, i.e., $\sum_{j \in U} b_{i j} x_{i j} \leq t_{i}, i=$ $1, \ldots, N_{B}$. From a practical perspective, it is also desirable for each user to be served by at most one BS. This is because, otherwise, coordination among multiple BSs is necessary, which is usually cumbersome in practice. The requirement that each user is served by at most one BS can be expressed as the constraint $\sum_{i \in B} x_{i j} \leq 1, j=1, \ldots, N_{U}$. When this constraint is enforced, the total number of users in the system can be expressed as $\sum_{i \in B} \sum_{j \in U} x_{i j}$. Note that a user $\hat{j} \in U$ that is not served by any BS will result in $x_{i \hat{j}}=0$ for every $i \in B$.

To maximize the number of users while ensuring efficient utilization of resources we consider a linear combination of these two quantities. In particular, let $\rho \in[0,1]$ be given, and let the objective be to maximize $\rho \sum_{i \in B} \sum_{j \in U} x_{i j}-(1-\rho) \sum_{i \in B} \sum_{j \in U} b_{i j} x_{i j}$. Increasing $\rho$ from 0 to 1 shifts the emphasis of the optimization from minimizing the number of resources used in the network to maximizing the number of users accommodated therein. As such, $\rho$ parametrizes a family of objectives, each with a corresponding number of users and a number of expended resources. To determine the optimal value of $\rho$, we follow a reasoning similar to the one in [9]. Doing so, it can be shown that any value of $\rho \in\left(\frac{\sum_{i \in B} t_{i}}{1+\sum_{i \in B} t_{i}}, 1\right)$ ensures maximizing the number of accommodated users and simultaneously minimizing the number of expended resources.

Assuming that $\rho$ lies within the aforementioned interval, the downlink user-to-BS association can be formulated as:

$$
\begin{aligned}
\max _{x_{i j}} \quad & \rho \sum_{i \in B} \sum_{j \in U} x_{i j}-(1-\rho) \sum_{i \in B} \sum_{j \in U} b_{i j} x_{i j} \\
& \sum_{j \in U_{i}} b_{i j} x_{i j} \leq t_{i}, \quad i \in B, \\
& \sum_{i \in B_{j}} x_{i j} \leq 1, \quad j \in U, \\
& x_{i j} \in\{0,1\}, \quad i \in B, j \in U_{i} .
\end{aligned}
$$


From (4), it can be readily verified that, apart from the binary constraint in $(4 \mathrm{~d})$, this formulation constitutes a convex linear optimization problem. However, the binary constraint makes this problem an instance of a non-convex mixed integer linear program (MILP). In fact, this problem is known as generalized assignment problem [10], which can be solved using various techniques, including those that use the branchand-bound approach and variations thereof. Unfortunately, the complexity of these algorithms grows exponentially with the number of variables and as such their applicability in the current scenario is questionable. To alleviate this difficulty, herein we consider utilizing an alternate technique for dealing with this class of problems, viz., the one based on semidefinite relaxation with randomization [7].

\section{User Association Via Semidefinite Relaxation}

Being an instance of the generalized assignment problem, the optimization of user-to-BS associations can be readily seen to be NP-hard. Hence, solving this problem optimally is computationally infeasible for all, but a relatively small class of HetNets with a limited number of users and BSs. To obtain resource-efficient user-to-BS associations, we propose to use the SDR technique with randomization. Unlike the techniques based on the branch-and-bound approach, SDR with randomization has polynomial complexity, which renders it suitable for solving large user-to-BS association problems that may arise in practice. In comparison with other polynomial complexity algorithms, SDR with randomization has provable approximation accuracy [11]. A somewhat similar technique for generating user-to-BS associations was developed in [12] in a different context. In particular, herein we consider maximizing the number of users served by a generic HetNet with an arbitrary number of macro and pico BSs per cell under QoS constraints, whereas in [12] the HetNet is restricted to have one macro and one pico BS per cell and the design objective is to maximize the total sum rate irrespective of the QoSs demanded by the users.

To cast (4) in a form that is more amenable to the optimization technique employed hereinafter, we will express the objective and the constraints using vector notation. Towards that end, let $X \in \mathbb{R}^{N_{B} \times N_{U}}$ and $B \in \mathbb{R}^{N_{B} \times N_{U}}$ be the matrices whose $i j$-th entries are $x_{i j}$ and $b_{i j}$, respectively. Let $D_{i} \in \mathbb{R}^{N_{B} \times N_{U}}$ be the all-zero matrix except its $i$-th column is equal to the $i$-th column of $B, i=1, \ldots, N_{B}$, and let $E_{j} \in \mathbb{R}^{N_{B} \times N_{U}}$ be the all-zero matrix except its $j$-th row is all ones, $j=1, \ldots, N_{u}$. Using $\operatorname{vec}(\cdot)$ to denote the operator that stacks the columns of a matrix on top of each other, we make the following definitions: $\mathbf{x} \triangleq \operatorname{vec}(X), \mathbf{b} \triangleq \operatorname{vec}(B)$, $\mathbf{d}_{i} \triangleq \operatorname{vec}\left(D_{i}\right)$ and $\mathbf{e}_{j} \triangleq \operatorname{vec}\left(E_{j}\right)$. Using this notation the problem in (4) can be expressed in the following form:

$$
\begin{array}{cl}
\max _{\mathbf{x}} & \rho \mathbf{1}^{T} \mathbf{x}-(1-\rho) \mathbf{b}^{T} \mathbf{x}, \\
\text { subject to } & \mathbf{d}_{i}^{T} \mathbf{x} \leq t_{i}, \quad i \in B, \\
& \mathbf{e}_{j}^{T} \mathbf{x} \leq 1, \quad j \in U, \\
& \mathbf{x} \in\{0,1\}^{N_{B} N_{U}},
\end{array}
$$

where $\mathbf{1} \in \mathbb{R}^{N_{U} N_{B}}$ is the all-one vector. To cast this problem in the standard form for SDR, the optimization variables must be antipodal rather than binary. Hence, we introduce the vector $\boldsymbol{\beta}=2 \mathbf{x}-\mathbf{1}$, which implies that $\boldsymbol{\beta} \in\{-1,1\}^{N_{B} N_{U}}$, and

$$
\mathbf{x}=\frac{1}{2}(\boldsymbol{\beta}+\mathbf{1}) \text {. }
$$

Using (6), the formulation in (5) can be cast as:

$$
\begin{array}{cl}
\max _{\mathbf{x}} & \frac{\rho}{2} \mathbf{1}^{T}(\boldsymbol{\beta}+\mathbf{1})-\frac{(1-\rho)}{2} \mathbf{b}^{T}(\boldsymbol{\beta}+\mathbf{1}), \\
\text { subject to } & \frac{1}{2} \mathbf{d}_{i}^{T}(\boldsymbol{\beta}+\mathbf{1}) \leq t_{i}, \quad i \in B, \\
& \frac{1}{2} \mathbf{e}_{j}^{T}(\boldsymbol{\beta}+\mathbf{1}) \leq 1, \quad j \in U, \\
& \boldsymbol{\beta} \in\{-1,1\}^{N_{B} N_{U} .}
\end{array}
$$

To use the SDR technique, the problem in (7) is expressed in a form in which the optimization variables are constrained to be in the cone of symmetric positive semidefinite matrices [7]. To do so, we define the following vectors in $\mathbb{R}^{N_{B} N_{U}+1}$, $\hat{\mathbf{b}} \triangleq\left[\begin{array}{ll}\mathbf{b}^{T} & \mathbf{b}^{T} \mathbf{1}\end{array}\right]^{T}, \hat{\mathbf{d}}_{i} \triangleq\left[\begin{array}{ll}\mathbf{d}_{i}^{T} & \mathbf{d}_{i}^{T} \mathbf{1}\end{array}\right]^{T}, i=1, \ldots, N_{B}$, $\hat{\mathbf{e}}_{j} \triangleq\left[\mathbf{e}_{j}^{T} \mathbf{e}_{j}^{T} \mathbf{1}\right]^{T}, j=1, \ldots, N_{U}, \hat{\mathbf{1}} \triangleq\left[\begin{array}{ll}\mathbf{1}^{T} & \mathbf{1}^{T} \mathbf{1}\end{array}\right]^{T}, \hat{\boldsymbol{\beta}} \triangleq$ $\left[\begin{array}{ll}\boldsymbol{\beta} & 1\end{array}\right]^{T}$ and $\hat{\mathbf{c}} \triangleq\left[\mathbf{0}_{N_{B} N_{U}}^{T} 1\right]^{T}$, where $\mathbf{0}_{N_{B} N_{U}}$ is the all-zero $N_{B} N_{U} \times 1$ vector. We also define the symmetric matrices $\boldsymbol{\Phi} \in \mathbb{R}^{N_{B} N_{U} \times N_{B} N_{U}}$ and $\boldsymbol{\Psi} \in \mathbb{R}^{N_{B} N_{U}+1 \times N_{B} N_{U}+1}$ to be $\boldsymbol{\Phi} \triangleq \boldsymbol{\beta} \boldsymbol{\beta}^{T}$ and $\boldsymbol{\Psi}=\hat{\boldsymbol{\beta}} \hat{\boldsymbol{\beta}}^{T}$; in particular, $\boldsymbol{\Psi}=\left[\begin{array}{cc}\boldsymbol{\Phi} & \boldsymbol{\beta} \\ \boldsymbol{\beta}^{T} & 1\end{array}\right]$. Finally, we define the $N_{B} N_{U}+1 \times N_{B} N_{U}+1$ rank-1 matrices $\mathbf{A}_{1} \triangleq \hat{\mathbf{c}} \hat{\mathbf{1}}^{T}, \mathbf{A}_{b} \triangleq \hat{\mathbf{c}} \hat{\mathbf{b}}^{T}, \mathbf{A}_{d_{i}} \triangleq \hat{\mathbf{c}} \hat{\mathbf{d}}_{i}^{T}, i=1, \ldots, N_{B}$ and $\mathbf{A}_{e_{j}} \triangleq \hat{\mathbf{c}} \hat{\mathbf{e}}_{j}^{T}, j=1, \ldots, N_{U}$. Using these definitions, it can be ready verified that the problem in (7) is equivalent to the following optimization problem.

$$
\begin{array}{ll}
\max _{\mathbf{\Psi}} & \frac{\rho}{2} \operatorname{Tr}\left(\mathbf{A}_{1} \boldsymbol{\Psi}\right)-\frac{1-\rho}{2} \operatorname{Tr}\left(\mathbf{A}_{b} \boldsymbol{\Psi}\right), \\
\text { subject to } & \frac{1}{2} \operatorname{Tr}\left(\mathbf{A}_{d_{i}} \mathbf{\Psi}\right) \leq t_{i}, \quad i \in B, \\
& \frac{1}{2} \operatorname{Tr}\left(\mathbf{A}_{e_{j}} \mathbf{\Psi}\right) \leq 1, \quad j \in U, \\
& \mathbf{\Psi} \succeq \mathbf{0}, \\
& \operatorname{diag}(\boldsymbol{\Psi})=1, \\
& \operatorname{rank}(\boldsymbol{\Psi})=1 .
\end{array}
$$

In this formulation, the binary constraint in $(7 \mathrm{~d})$ is replaced with the equivalent constraints in (8e) and (8f). The constraint in $(8 \mathrm{e})$ is linear and therefore can be readily incorporated in the optimization framework. However, the rank-1 constraint in (8f) is highly non-convex and, in fact, it is this constraint that captures the NP-hardness of the original problem in (7). Despite being ostensibly more complex than (7), the formulation in (8) offers the advantage of being readily amenable to the SDR technique, which we describe next.

\section{A. SDR with Gaussian Randomization}

Our main contribution is to show that maximizing the number of users in a generic HetNet with a given set of available resources can be cast in the form in (8). This form is amenable 
to the standard SDR with Gaussian randomization approach [7]. For completeness, this approach will be tailored below to provide an approximate solution of (8).

The main difficulty of solving (8) follows from the nonconvexity of the rank-1 constraint. By dropping this constraint, the resulting problem can be seen to be linear over the set of matrices $\boldsymbol{\Psi}$ that lie in the positive semidefinite cone. In other words, the relaxation induced by dropping the rank-1 constraint yields an efficiently solvable convex optimization problem in the form of a semidefinite program. This program forms the basis of the solution approach adopted herein.

Let $\mathbf{z}, \mathbf{Z}$ and $\mathbf{R}$ be the optimization variables of the relaxed problem corresponding to $\boldsymbol{\beta}, \boldsymbol{\Phi}$ and $\boldsymbol{\Psi}$ in the original problem in (8), respectively. The convexity of the relaxed version of (8) implies that its global optimal solution, $\mathbf{z}^{*}, \mathbf{Z}^{*}$ and $\mathbf{R}^{*}$, can be readily obtained. Since the difference between (8) and its relaxed version is the rank-1 constraint, it can be readily seen that if the rank of the matrix generated by the relaxed program is 1 , i.e., $\operatorname{rank}\left(\mathbf{R}^{*}\right)=1$, the vector $\mathbf{z}^{*}$ yielded by solving the relaxed version of (8) is binary and constitutes the optimal user-to-BS association. However, the solution of the relaxed problem is in general not rank-1. In this case, the Gaussian randomization approach can be used to obtain a desirable user-to-BS association for the problem in (7).

In the Gaussian randomization approach, a stochastic version of the relaxation of (8) is conceived. In that version, the vector $\mathbf{z}^{*}$ generated by solving the relaxed program is considered as the mean of a multivariate Gaussian $N_{B} N_{U}$-dimensional random vector, and $\mathbf{Z}^{*}$ and $\mathbf{Z}^{*}-\mathbf{z}^{*} \mathbf{z}^{* T}$ are considered as the correlation and covariance matrices of this random vector, respectively. In particular, let $\Delta$ be a set of $J$ random vector samples drawn from the Gaussian distribution with mean $\mathbf{z}^{*}$ and covariance $\mathbf{Z}^{*}-\mathbf{z}^{*} \mathbf{z}^{* T}$, i.e., $\boldsymbol{\Delta}=\left\{\boldsymbol{\delta}^{j}\right\}_{j=1}^{J}, \boldsymbol{\delta}^{j} \sim \mathcal{N}\left(\mathbf{z}^{*}, \mathbf{Z}^{*}-\right.$ $\left.\mathbf{z}^{*} \mathbf{z}^{* T}\right), j=1, \ldots, J$. Let $\hat{\boldsymbol{\delta}}=\left[\begin{array}{ll}\boldsymbol{\delta}^{T} & 1\end{array}\right]^{T}$ and $\hat{\mathbf{z}}^{*}=\left[\begin{array}{ll}\mathbf{z}^{* T} & 1\end{array}\right]^{T}$. Hence, the vectors in $\Delta$ can be seen to provide an approximate solution to the following stochastic optimization problem:

$$
\begin{array}{ll}
\underset{\mathbb{E}\left\{\hat{\boldsymbol{\delta}} \hat{\boldsymbol{\delta}}^{T}\right\}=\mathbf{R}^{*}, \mathbb{E}\{\hat{\boldsymbol{\delta}}\}=\hat{\mathbf{z}}^{*}}{ } & \mathbb{E}\left\{\hat{\boldsymbol{\delta}}^{T}\left(\frac{\rho}{2} \mathbf{A}_{1}-\frac{1-\rho}{2} \mathbf{A}_{b}\right) \hat{\boldsymbol{\delta}}\right\}, \\
\text { subject to } & \mathbb{E}\left\{\hat{\boldsymbol{\delta}}^{T} \mathbf{A}_{d_{i}} \hat{\boldsymbol{\delta}}\right\} \leq t_{i}, \quad i \in B, \\
& \mathbb{E}\left\{\hat{\boldsymbol{\delta}}^{T} \mathbf{A}_{e_{j}} \hat{\boldsymbol{\delta}}\right\} \leq 1, \quad j \in U . \\
& \mathbb{E}\left\{\hat{\boldsymbol{\delta}}_{r}^{2}\right\}=1, r=1, \ldots, N M .
\end{array}
$$

Notice that because the matrix $\mathbf{R}^{*}=\left[\begin{array}{cc}\mathbf{Z}^{*} & \mathbf{z}^{*} \\ \mathbf{z}^{* T} & 1\end{array}\right]$ generated by the relaxed version of (8) is positive semidefinite, its Schur complement, $\mathbf{Z}^{*}-\mathbf{z}^{*} \mathbf{z}^{* T}$, is also positive semidefinite. In other words, $\mathbf{Z}^{*}-\mathbf{z}^{*} \mathbf{z}^{* T}$ constitutes a valid covariance matrix of the random vectors $\left\{\boldsymbol{\delta}^{j}\right\}$. This establishes the equivalence between (9) and the relaxed version of (8).

Now, the vectors in $\boldsymbol{\Delta}$ are used to find candidate binary solutions $\left\{\tilde{\boldsymbol{\beta}}^{j}\right\}_{j=1}^{J}$ for the problem in (7) by quantizing the entries of each realization of $\left\{\boldsymbol{\delta}^{j}\right\}_{j=1}^{J}$. In particular,

$$
\tilde{\boldsymbol{\beta}}^{j}=\operatorname{sgn}\left(\boldsymbol{\delta}^{j}\right), \quad j=1, \ldots, J,
$$

where $\operatorname{sgn}(\cdot)$ is the element-wise signum function. Using $\tilde{\boldsymbol{\beta}}^{j}$ and (6), we obtain candidate solutions of (5), $\tilde{\mathbf{x}}^{j}$. The candidate that yields the largest objective and satisfies the constraints in (5) is used for associating the users to the BSs, i.e.,

$$
\mathbf{x}^{*}=\arg \max _{\mathcal{F}_{j}} \tilde{\mathbf{x}}^{j}, \quad \mathcal{F}_{j} \triangleq\left\{\tilde{x}^{j}: \tilde{x}^{j} \text { satisfying (5b), (5c) }\right\} .
$$

\section{PERformance eVAluation}

In this section, we evaluate the performance of the proposed user-to-BS association method through numerical comparisons with two popular user-to-BS association algorithms, viz., the max-SINR algorithm and the RE algorithm [13]. We consider a two-tier HetNet scenario consisting of one macro and three pico BSs. The transmit powers of macro and pico BSs are 46 $\mathrm{dBm}$ and $35 \mathrm{dBm}$, respectively. The location of the macro BS is assumed to be fixed, whereas the locations of the pico BSs and the users are assumed to be random and uniformly distributed on a $500 \mathrm{~m} \times 500 \mathrm{~m}$ square. For the propagation environment, shadowing is assumed to have a log-normal distribution with a standard deviation of $\sigma_{s}=8 \mathrm{~dB}$ and the path loss between the BSs and users is assumed to be $L(d)=34+40 \log (d)$, where $d$ is the distance between users and BSs in meters. The noise power at all receivers is assumed to be $-104 \mathrm{dBm}$. For all numerical simulations, the results are averaged over 50 independent channel realizations. For practical considerations, users are allocated integer numbers of RBs; fractional RB allocation not allowed. The bandwidth of each $\mathrm{RB}$ is set to be $180 \mathrm{kHz}$ [8]. The SDR programs are solved using the CVX package [14] with SDPT3 solver. The number of Gaussian samples used in the randomization phase is set to be $J=100$ for each channel realization.

\section{Example 1}

In this example we consider the scenario in which the users accessing the network, each has a QoS demand of $Q_{j}=500$ kilo-bits-per-second (kbps), $j \in U$, and each BS has a total of $t_{i}=50$ available RBs, for $i \in B$. The user-to-BS associations are generated using the max-SINR, the RE and the proposed SDR-based algorithms. In Figure 2 the number of users that could be accommodated in the network is plotted versus the total number of users who wish to use the network. For the $\mathrm{RE}$ algorithm, the biasing parameter for each BS was set to be either $5 \mathrm{~dB}$ or $10 \mathrm{~dB}$ [13].

From this figure, it can be seen that the proposed SDRbased algorithm outperforms both the max-SNR and the RE algorithms over the entire range of available network users. This is especially true when the system experiences high loading conditions. When the system is lightly loaded, all algorithms can accommodate a high percentage of the available users, and when the system is heavily loaded, the available RBs are exhausted and only a small percentage of users can be accommodated. For instance, when the total number of users is 100 , the proposed algorithm can accommodate the demands of about $17 \%$ more users than the RE algorithms and about $34 \%$ more users than the max-SINR algorithm. 


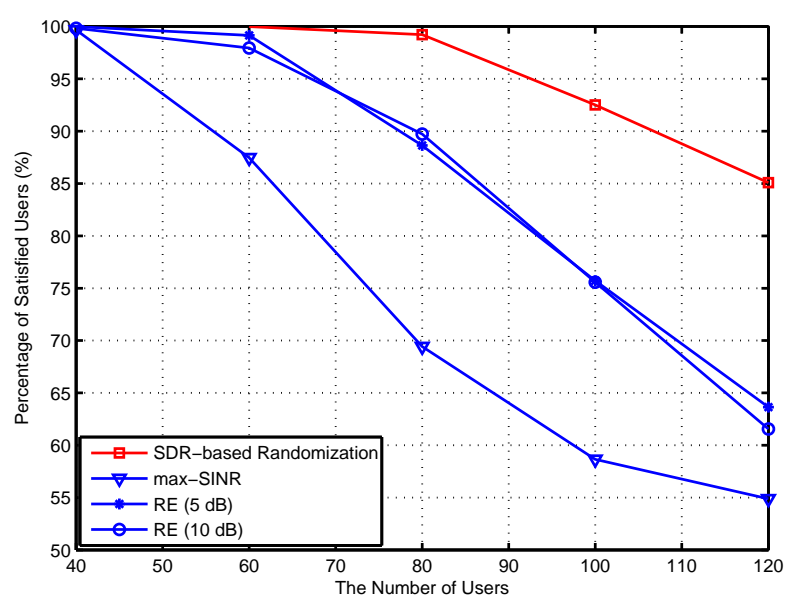

Fig. 2. Percentage of satisfied users versus the total number of user accessing the network.

\section{Example 2}

In this example we investigate the scenario in which the total number of users is $N_{U}=100$, and each BS has a total of $t_{i}=50$ available RBs, for $i \in B$. Similar to Example 1, we consider the user-to-BS associations generated using the max-SINR, the RE and the proposed SDR-based algorithms. However, in this example, we compare the performance of these algorithms with the increase in the QoS requirements. This comparison is depicted in Figure 3.

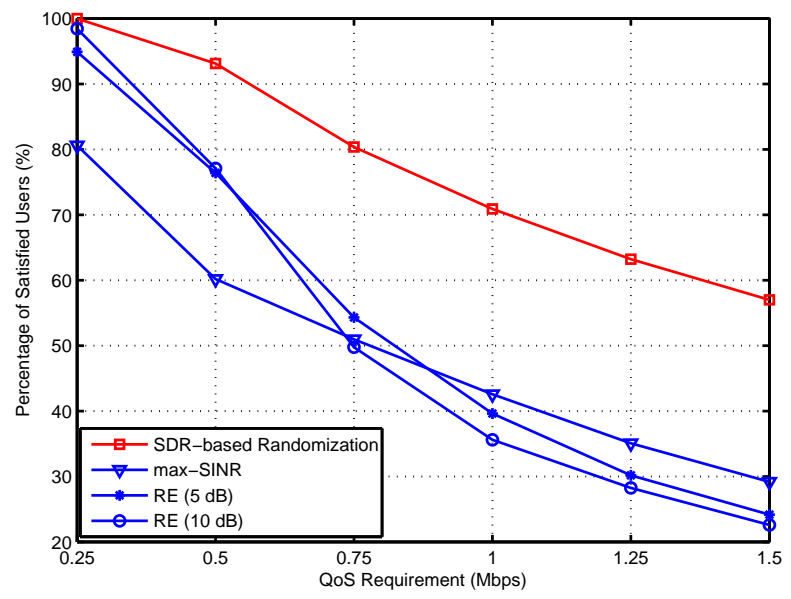

Fig. 3. Percentage of satisfied users versus QoS requirements.

From Figure 3, it can be seen that the proposed SDR-based algorithm performs better than both the max-SINR and the RE algorithms over the entire range of QoS requirements. However, its advantage over these algorithms is more pronounced at high QoS requirements. For instance, when the users' QoS requirement is $1000 \mathrm{kbps}$, the proposed algorithm can accommodate the demands of about $32 \%$ more users than the $\mathrm{RE}$ algorithm with the biasing parameter of $5 \mathrm{~dB}$, about $36 \%$ more users than the RE algorithm with the biasing parameter of $10 \mathrm{~dB}$, and about $29 \%$ more users than the max-SINR algorithm. At low QoSs, all algorithms can accommodate a large percentage of users, and at high QoSs, the number of available of RBs becomes the main bottleneck, thereby limiting the performance of the three algorithms.

\section{CONCLUSION}

This paper investigated the problem of associating users to BSs in a resource-limited downlink scenario of a multi-tier HetNet. In this scenario, the users may have disparate QoS demands and the BSs may have disparate powers and disparate number of available RBs. We proposed a QoS-guaranteed user-to-BS association algorithm based on SDR and Gaussian randomization. This algorithm has polynomial complexity and provable approximation accuracy. In comparison with currently available techniques, the proposed algorithm enables the accommodation of more users with less resources.

\section{REFERENCES}

[1] A. Damnjanovic, J. Montojo, J. Cho, H. Ji, J. Yang, and P. Zong, "UE's role in LTE-Advanced heterogeneous networks," IEEE Commun. Mag., vol. 50, pp. 164-176, Feb. 2012.

[2] C. Foster, I. Dickie, G. Maile, H. Simth, and M. Crisp, "Understanding the environmental impact of communication systems," Ofcom Final Report, Dec. 2009.

[3] R. Madan, J. Borran, A. Sampath, N. Bhushan, A. Khandekar, and T. Ji, "Cell association and interference coordination in heterogeneous LTE-A cellular networks," IEEE J. Select. Areas Commun., vol. 28, pp. 1479-1489, Dec. 2010.

[4] I. Guvenc, M.-R. Jeong, I. Demirdogen, B. Kecicioglu, and F. Watanabe, "Range expansion and inter-cell interference coordination (ICIC) for picocell networks," in Proc. IEEE Veh. Tech. Conf. (VTC-Fall), pp. 1-6, Sept. 2011.

[5] Q. Ye, B. Rong, Y. Chen, M. Al-Shalash, C. Caramanis, and J. G. Andrews, "User association for load balancing in heterogeneous cellular networks," IEEE Trans. Wireless Commun., vol. 12, pp. 2706-2716, June 2013.

[6] K. Shen and W. Yu, "Distributed pricing-based user association for downlink heterogeneous cellular networks," IEEE J. Select. Areas Commun., vol. 32, pp. 1100-1113, June 2014.

[7] Z.-Q. Luo, W.-K. Ma, A. M.-C. So, Y. Ye, and S. Zhang, "Semidefinite relaxation of quadratic optimization problems," IEEE Signal Process. Mag., vol. 27, pp. 20-34, May 2010.

[8] 3GPP, "Evolve universal terrestrial radio access (E-UTRA) and evolved universal terrestrial radio access network (E-UTRAN)," 3GPP, TS 36.300 v11.7.0, 2013

[9] E. Matskani, N. D. Sidiropoulos, Z.-Q. Luo, and L. Tassiulas, "Convex approximation techniques for joint multiuser downlink beamforming and admission control," IEEE Trans. Wireless Commun., vol. 7, pp. 2682 2693, 2008.

[10] D. Morales and H. Romeijn, "The generalized assignment problem and extensions," in Handbook of Combinatorial Optimization (D.-Z. Du and P. Pardalos, eds.), pp. 259-311, Springer US, 2005.

[11] M. X. Goemans and D. P. Williamson, "Improved approximation algorithms for maximum cut and satisfiability problems using semidefinite programming," J. ACM, vol. 42, pp. 1115-1145, Nov. 1995.

[12] S. Corroy and R. Mathar, "Semidefinite relaxation and randomization for dynamic cell association in heterogeneous networks," in Proc. IEEE Glob. Commun. Conf. (Globecom), pp. 2373-2378, Dec. 2012.

[13] "On cell range expansion-R1-105294," in The Mobile Broadband Standard-3GPP Meeting R1-62b, Texas Instruments, Oct. 2010. Available at: http://www.3gpp.org/DynaReport/TDocExMtg--R1-62b--28041. htm.

[14] M. Grant and S. Boyd, "CVX: Matlab software for disciplined convex programming, version 2.1," Mar. 2014. Available at:http://cvxr.com/cvx. 